\title{
Active Target System with MPPC Readout for Hyperon-Proton Scattering Experiment
}

\author{
Koji Miwa ${ }^{* \dagger}$ Ryotaro Honda, Kenji Hosomi, Takeshi Koike, Yue Ma, \\ Tomokazu Otani, Misako Sato, Kotaro Shirotori, Hirokazu Tamura, \\ Takeshi Yamamoto, Yoshio Yonemoto \\ Tohoku University
}

\begin{abstract}
We are developing an active target system with Multi-Pixeled Photon Counter (MPPC) readout for a hyperon-proton (YN) scattering experiment. The active target consists of scintillation fibers and each scintillation fiber is read by one MPPC. In order to check the detector response to a minimum ionization particle (MIP) and to a low energy proton, three test experiments with different beam conditions were performed. The light yield for the $450 \mathrm{MeV}$ positron beam was obtaind to be about 7 photons (fired pixel), and the trajectory of the positron was clearly identified. The response to a low-energy proton was checked using the proton beam of energies from $10 \mathrm{MeV}$ to $50 \mathrm{MeV}$. The light yields were about 110 and 270 photons for protons penetrating through the fiber layers and stopped, respectively. The range of the proton in the active target corresponds to the energy of the proton. Finally, as the feasibility test of the YN scattering experiments, we have performed a proton scattering experiment using our imaging system. This system worked well under a rather high counting rate of about $50 \mathrm{kHz}$. The images of the proton-proton and proton- ${ }^{12} \mathrm{C}$ scattering events were clearly identified.
\end{abstract}

International Workshop on New Photon Detectors PD09

June 24-26 2009

Shinshu University Matsumoto Japan

\footnotetext{
*Speaker.

${ }^{\dagger}$ E-mail: miwa9@lambda.phys.tohoku.ac.jp
} 


\section{Introduction of Hyperon Nucleon Scattering Experiment}

A hyperon-nucleon $(\mathrm{YN})$ scattering experiment is the most direct way to understand the $\mathrm{YN}$ interaction, just as the nuclear (NN) force has been understood based on the abundant data of NN scattering. It is important to understand nuclear force from a quark level by introducing a new degree of freedom, "strangeness". Deep understanding of the one-boson exchange model can be obtained by including the YN interaction because new meson exchanges such as $K$ and $K^{*}$ play an important role [1]. In a quark picture, by introducing strange quarks, interesting spin and isospin configurations, which is absent in the $\mathrm{NN}$ force, can appear. For example, in a $\Sigma^{+} p$ system, there is an isospin $(I)$-spin $(S)$ configuration of $(I, S)=(3 / 2,1)$ where two quarks must be in the identical state and the large repulsive core due to the quark Pauli blocking is predicted [2] .

From experimental viewpoints, however, the $\mathrm{YN}$ scattering experiment is difficult due to the short life time of hyperons, and the data of the YN scatterings are quite poor. In order to perform the YN scattering experiment, a special experimental technique should be developed. The upgrade of a high speed imaging detector, which was used in the KEK experiment, is one possibility. In the KEK-PS, the YN scattering experiments (KEK-PS E289, E452) have been carried out with active targets using a Scintillation Fiber (Scifi) and a bulk Scintillator (SCITIC) [3, 4]. In the E289 experiment, the trajectories of the hyperon and scattered proton were detected as images using the Scifi and Image Intensifier Tube (IIT) for its readout. While the feasibility of using the active target was demonstrated, the number of observed events was about 30, which is not sufficient. The reason for this poor statistics was a slow operation of IIT. At J-PARC, we want to perform a high statistics experiment using kaon or pion beams of the intensity of more than $1 \%$ /spill. Now we are developing a high speed Scifi target system with a Multi-pixeled Photon Counter (MPPC) readout. The advantages of using MPPC are the followings.

- The time response is fast $(<10 \mathrm{~ns})$, and MPPC can be operated under a high intensity beam.

- Gain is large $\left(10^{5} \sim 10^{6}\right)$, and MPPC can detect one photon.

- MPPC can be operated in a magnetic field, and it can be placed inside a magnetic spectrometer.

- MPPC can trigger a reaction event using a pulse height information.

The detailed information about the future experiment can be found in the reference [5]. The responses to a minimum ionization particle (MIP) and a slow proton should be checked. We made a small prototype detector using 20 50 scintillation fibers and MPPC readouts, and the positron and proton beams were irradiated to the prototype detector. As the feasibility test of the YN scattering experiment, we also performed a proton scattering experiment using our imaging system. In this contribution, we will report the results of these test experiments.

\section{The Test Experiment with a $450 \mathrm{MeV} e^{+}$Beam}

Basic requirements on the active target system for the $\mathrm{YN}$ scattering experiment are two, namely (1) to detect a MIP and (2) that MPPC can be operated in a magnetic field. In order to 
evaluate these aspects, we performed a test experiment with $450 \mathrm{MeV} e^{+}$beam provided at the Laboratory of Nuclear Science (LNS)-Tohoku. We made a Scifi bundle of 5 segment and 4 layer configuration. We used 20 scintillation fibers (KURARAY SCSF-78SJ) whose square cross section was $1 \mathrm{~mm} \times 1 \mathrm{~mm}$. Hamamatsu MPPC (S10362-11-050C) with effective area of $1 \mathrm{~mm} \times 1 \mathrm{~mm}$ was used. The effective area consisted of 400 pixels of APD whose size was $50 \mu \mathrm{m} \times 50 \mu \mathrm{m}$. The signal from MPPC was amplified by ORTEC Fast Amplifier (FTA 820). The Scifi and MPPC were placed in a gap of a magnet. The magnetic field was changed from $0 \mathrm{~T}$ to $1.06 \mathrm{~T}$ for the testing of the MPPC performance in magnetic fields.

Figure 1 shows the photon number detected by Scifi-MPPC system for the $450 \mathrm{MeV} e^{+}$beam (no magnetic field). The mean photon number was $\sim 7.8$, which was a sufficient value. When more than 2 photons were demanded, the efficiency of detecting MIP was 95\%. We confirmed that the photon number and the gain of MPPC did not change in the magnetic fields which was changed from $0 \mathrm{~T}$ to $1.06 \mathrm{~T}$. Figure 2 shows images of the 5 segment and 4 layer Scifi when the $e^{+}$beam passed through it. The trajectory of the $e^{+}$beam is clearly identified.

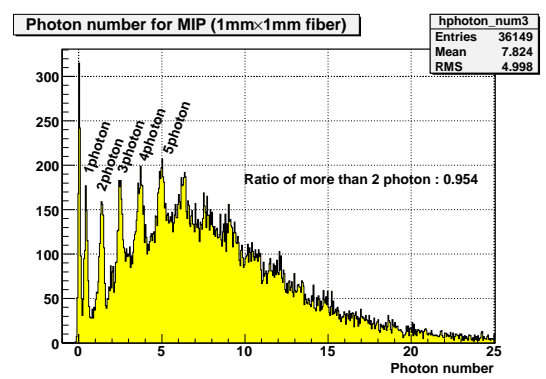

Figure 1: Detected photon number by a scintillation fiber and MPPC system when a $e^{+}$beam passed through the fiber (no magnetic field). Each peak corresponds to the photon numbers detected by MPPC.
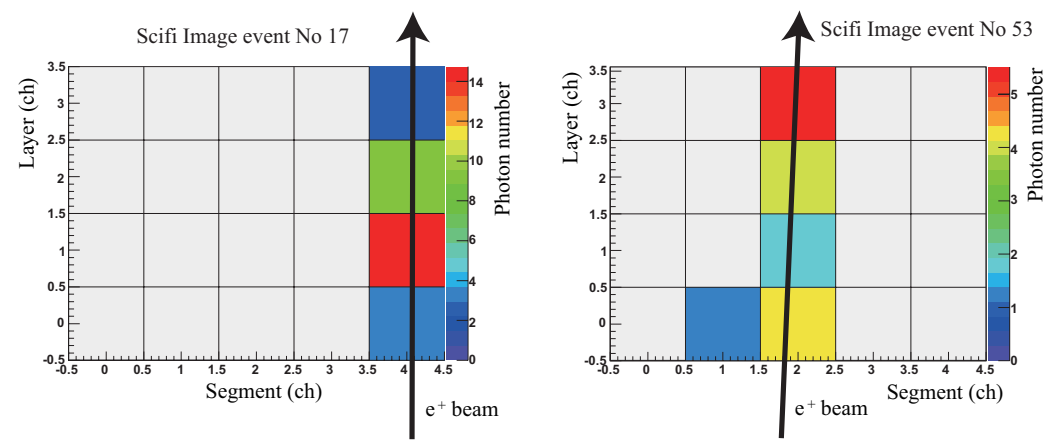

Figure 2: Images of 5 segment and 4 layer Scifi when $e^{+}$beam passed through the Scifi.

\section{The Test Experiment with a Proton Beam}

The test experiment using a proton beam of the energy from $10 \mathrm{MeV}$ to $50 \mathrm{MeV}$ has been performed at the Cyclotron Radio Isotope Center (CYRIC) facility in Tohoku university. The 
purpose of the experiment was to check the light yield and the dynamic range of MPPC for a slow proton. The total energy deposit of a particle which is stopped inside the active target should be measured from the range. Therefore we checked the relation between the energy of the proton beam and its range inside the target by changing the energy of the proton beam.

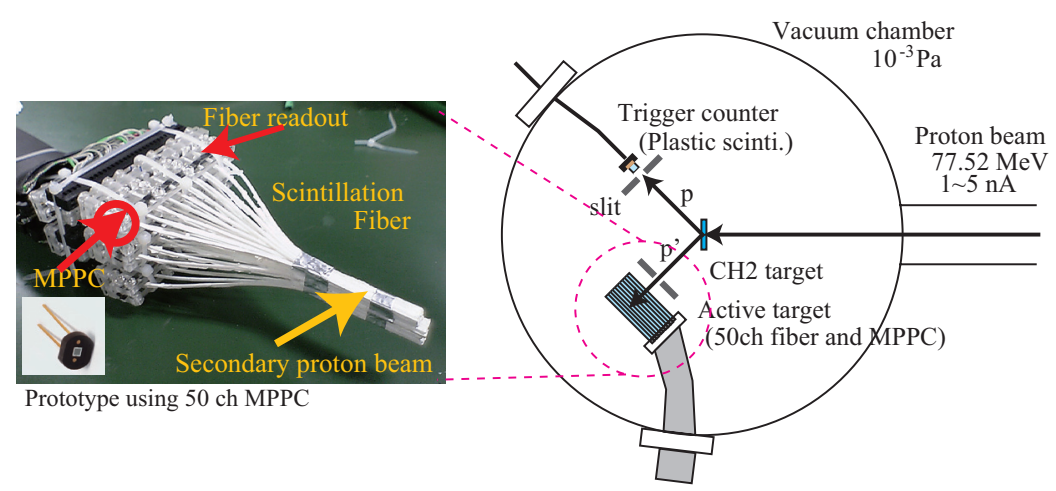

Figure 3: The schematic view of the experimental setup. The left picture shows the Scifi active target made by 50 scintillation fibers. In each fiber, MPPC was connected at the one side. This active target was located inside a vacuum chamber. The secondary proton beam produced in the pp scattering at the $\mathrm{CH}_{2}$ target was irradiated to the active target. The pp scattering was tagged by the coincidence of the trigger counter and 1st layer of the active target. The energy and intensity of the primary proton were $77.52 \mathrm{MeV}$ and $1 \sim 5 \mathrm{nA}$, respectively. The vacuum in the chamber was $10^{-3} \mathrm{~Pa}$.

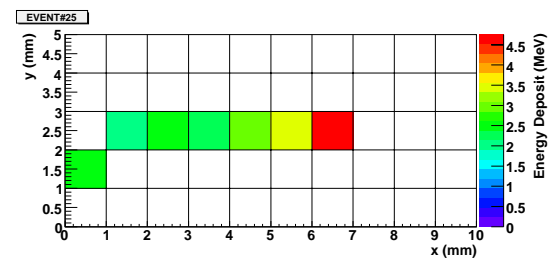

Figure 4: Typical event display of the $30 \mathrm{MeV}$ proton. Each pixel corresponds to the scintillation fiber and the color corresponds to the energy deposit in the fiber. The proton stops at the sevenths layer and Bragg curve of $d E / d x$ can be recognized.

The schematic view of the experimental setup is shown in Figure 3. The proton beam of 77.52 $\mathrm{MeV}$ was used as a primary beam. The intensity was set to be $1 \sim 5 \mathrm{nA}$. In order to irradiate various energies of proton beams, we used proton-proton scattering events at the polyethylene $\left(\mathrm{CH}_{2}\right)$ primary target which were selected by taking a coincidence between a trigger counter and the first layer of the active target. By changing the angles of these detectors, proton beams from $10 \mathrm{MeV}$ to $50 \mathrm{MeV}$ were irradiated to the active target. The energy spread of the secondary proton beam was determined by the size of a slit $(\phi 3 \mathrm{~mm})$ in front of the trigger counter, and it was expected to be $\pm 1 \mathrm{MeV}$. We made an active target of the 5 segment and 10 layer configuration Scifi bundle with 50 channels of MPPC readouts as shown in the left picture in Figure 3. Figure 4 shows a typical image of the $30 \mathrm{MeV}$ proton beam which is stopped at the seventh layer. The color of each pixel corresponds to the energy deposit in each fiber. A typical Bragg curve showing the 
variation of $d E / d x$ as a function of the penetration depth in Scifi can be seen. The active target and the trigger counter were installed in a vacuum chamber at $10^{-3} \mathrm{~Pa}$.

At first, we discuss the response of each MPPC. The left and right figures in Figure 5 show the ADC spectra for the protons penetrating through the fiber and the protons stopped in the fiber, respectively. The upper axis corresponds to the calculated energy deposit. For the energy calibration, we irradiated $60 \mathrm{MeV}$ proton beam which penetrated all layers of the active target. The obtained peak channel of each fiber was matched to the energy deposit calculated by a Monte Carlo simulation. Because the ADC gain for a single photon was measured to be $\sim 10 \mathrm{ch}$, the numbers of detected photons corresponded to 110 and 270 photons for the penetrating proton and stopped proton, respectively. From the previous experiment using the positron beam, the photon number for MIP partile is obtained to be $\sim 7$. The ADC channel for MIP particle corresponds to $\sim 70 \mathrm{ch}$ as shown by a hatched region in Figure 5. Therefore the signal from the slow proton or hyperon can be separated from the MIP particle and we can use the information of the energy deposit as a trigger to select a reaction event.

The left figure in Figure 6 shows the stopping layer for seven different energy proton beams. The range is consistent with the beam energy. The spread of the stopping layer can be explained by the spread of energy of proton beam. The right figure in Figure 6 shows the total energy deposit which is the sum of the energy deposit of all hit fibers. The tendency of the total energy deposit is consistent with the energy of proton. However the calculated value of the energy deposit and the incident beam energy is different. One of the possible reason is due to the saturation of the fired pixel. But this is not understood yet.
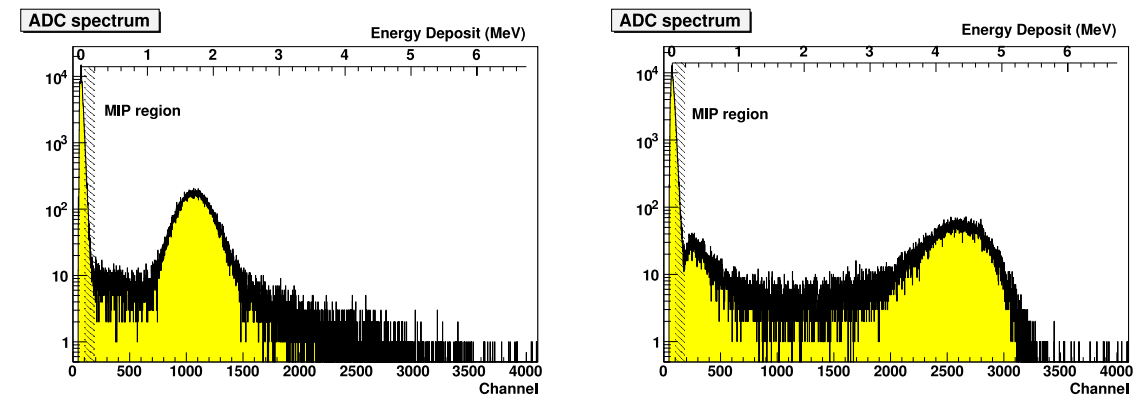

Figure 5: ADC spectra of one MPPC channel. The left and right figures show the ADC spectra for the penetrating and stopped protons, respectively. The upper axis represents the calculated energy deposit. The hatched region corresponds to the energy deposit for the MIP particles.

\section{The $p p$ and $p^{12} \mathrm{C}$ Scattering Experiments Using The Prototype Detector}

Finally we report our test experiment to measure the proton double scattering experiment to investigate the feasibility of the $\mathrm{YN}$ scattering experiment. By using the prototype detector consisted of $(\mathrm{CH})_{n}$, the $p p$ and $p^{12} \mathrm{C}$ scattering cross sections should be measured. We performed a proton double scattering experiment as shown in Figure 7. The secondary proton beam produced by the $p^{12} \mathrm{C}$ scattering at the primary ${ }^{12} \mathrm{C}$ target was irradiated to the active target made from 6 segment $\times 6$ layer fibers. The active target was surrounded by trigger counters which were plastic 

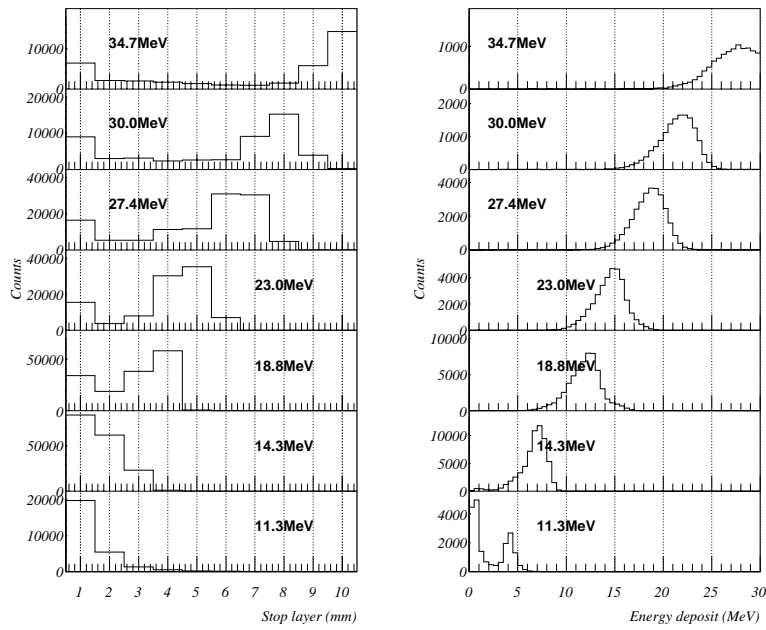

Figure 6: The stop layer distribution (left figure) and total energy deposit distribution (right figure) for various energy proton beams. The range is consistent with the beam energy. The total energy deposit is the sum of the energy deposit of all hit fibers.

scintillators read by MPPC in order to trigger the scattering events. In addition to the cross section measurement, we also tried to measure the left-right asymmetry in $p^{12} \mathrm{C}$ scattering, because ${ }^{12} \mathrm{C}$ has a high analyzing power of 0.95 for $60 \sim 80 \mathrm{MeV}$ proton beam.

The intensity of primary beam was $\sim 10^{11} \mathrm{~Hz}(\sim 100 \mathrm{nA})$ and the intensity of the secondary beam was $\sim 50 \mathrm{kHz}$. The left and right figures in Figure 8 show the typical event displays of the $p p$ and $p^{12} \mathrm{C}$ scatterings, respectively. This system worked well under a rather high counting rate and the images of the scattering events were clearly identified. Now the analysis of cross section measurement is in progress.

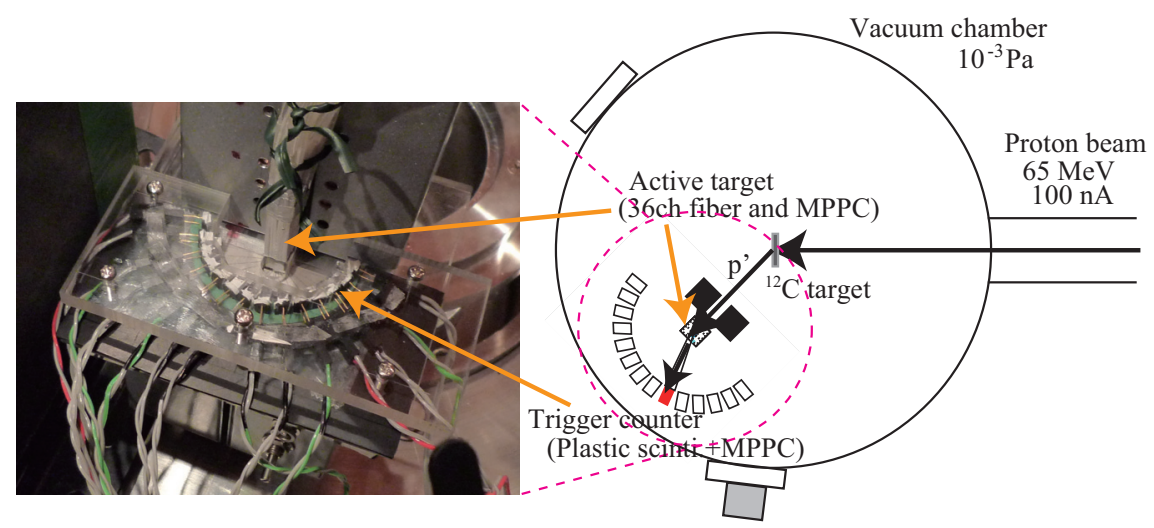

Figure 7: The schematic view of the experimental setup of the $p p$ and $p{ }^{12} \mathrm{C}$ scattering experiment. The active target was made by 36 channel fibers of 6 segment and 6 layer configuration. The active target was surrounded by the trigger counters to tag the scattering event in the active target. The energy and intensity of the primary proton were $65 \mathrm{MeV}$ and $\sim 100 \mathrm{nA}$, respectively. The vacuum in the chamber was $10^{-3} \mathrm{~Pa}$. 


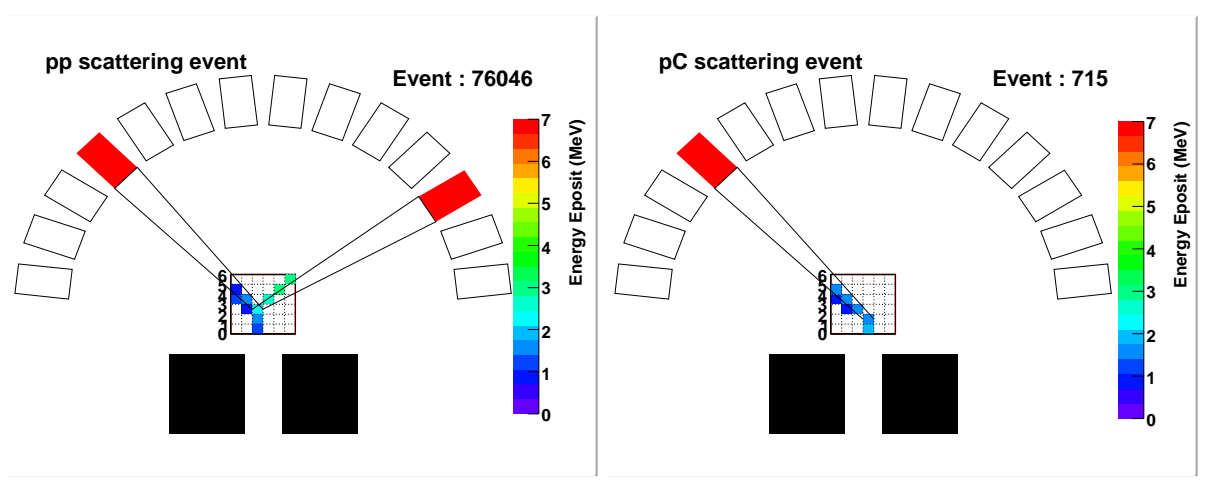

Figure 8: The left and right figures show the typical event displays of $p p$ and $p^{12} \mathrm{C}$ scattering events, respectively. In the $p p$ scattering event, two particles are detected by the detector. On the other hand, in the $p^{12} \mathrm{C}$ scattering, only one particle is scattered at the vertex.

\section{Summary}

We are developing an active target system with Multi-Pixeled Photon Counter (MPPC) readout for hyperon-proton scattering experiment. The responses of the detector to MIP and the low energy proton were checked through three kinds of test experiments. The light yield for the MIP particle was obtained to be about 7 photons (fired pixel). The light yields for the penetrating proton and the stopped proton were obtained to be about 110 and 270 photons, respectively. The range of the proton in the active target corresponded to the energy of the proton beam. Finally, as the feasibility test of $\mathrm{YN}$ scattering experiments, we have performed a proton scattering experiment using our imaging system. This system worked well under a rather high counting rate and images of protonproton and proton- ${ }^{12} \mathrm{C}$ scattering events were clearly identified.

A radiation damage of MPPC might be a serious problem in the YN scattering experiment. We do not yet perform a quantitative study of MPPC for radiation hardness, which remains to be studied in the future.

We would like to express our thanks to staffs of LNS-Tohoku and CYRIC for providing high quality beams during the experiment. We also acknowledge N. Chiga for his technical supports. This work was supported by dean's Grant for Exploratory Research (Graduate School of Science at Tohoku university).

\section{References}

[1] M.M. Nagels et al., Phys. Rev. D15 (1977) 2547; D20 (1979) 1633

[2] Y. Fujiwara, C. Nakamoto, Y. Suzuki, Prog. Theor. Phys. 94 (1995) 214; 94 (1995) 353; Phys. Rev. Lett. 76 (1996) 2242

[3] Y. Kondo et al., Nucle. Phys. A676 (2000) 371

[4] H. Kanda et al., Nucle. Phys. A761 (2005) 41

[5] Letter of Intent for an experiment at J-PARC, http://j-parc.jp/NuclPart/pac_0801/pdf/LOI_Miwa_YN.pdf 